Krzysztof Kogut*

\title{
Semi-active Suspension System Modelling and Parameters Identification
}

\section{Introduction}

Automotive vehicle suspension has been a subject of research interest both in academia and industry for many years. Modern car suspension systems can be divided into three groups: passive, semi-active and active. Most conventional cars are equipped with the first group (composed of springs and fixed dampers). Semi-active suspensions differ from passive suspensions in that they utilize shock absorbers with variable stiffness. The ability to change damper characteristics according to the road profile improves ride quality. Lastly, active suspension uses an actuator placed between the sprung body mass and the unsprung wheel mass exerting an independent force on the system of suspension. This solution offers a significant improvement of road handling and provides passengers with optimized ride comfort. The disadvantage of the active suspension in comparison to the semi-active is, among other things, its complexity and high power consumption. On the other hand, semiactive systems are unable to generate control force in the direction opposite to the current speed of the suspension (they can only switch the viscous damping coefficient of the shock absorber). However, MR brakes have been recently used in variuos applications, including vibration control in the suspensions of cars or trains.

In this study emphasis was placed on the modelling of a laboratory semi-active suspension system $\left(\mathrm{SAS}^{1}\right)$ depicted in Figure 1. Generally, literature devoted to car suspension modelling considers a two degree of freedom (DOF) quarter-car model, a six and a infinite DOF full car model [9]. The investigated device is modelled as a nonlinear 2DOF mass coupled system with a magnetorheological (MR) rotary damper. The parameter estimation technique described in this paper is based on the Grey-box model identification technique $[1,2]$. In this approach, the physical model of the system is utilized and its parameters are being identified through the comparison of the modelled and experimental trajectories [7].

\footnotetext{
* AGH University ofcience and Technology, Krakow, Poland

1 The device is manufactured by INTECO Sp. z o.o.
} 
This paper is organized as follows. In section 2, the SAS device is introduced. The dynamics of a suspension system consisting of a spring and MR damper are explained along with model parameters identification in section 3.1. Finally, the description of the car wheel, the eccentricity (road profile generator) and an estimation of the missing full model parameters is presented in section 3.2. Final remarks can be found in section 4 .

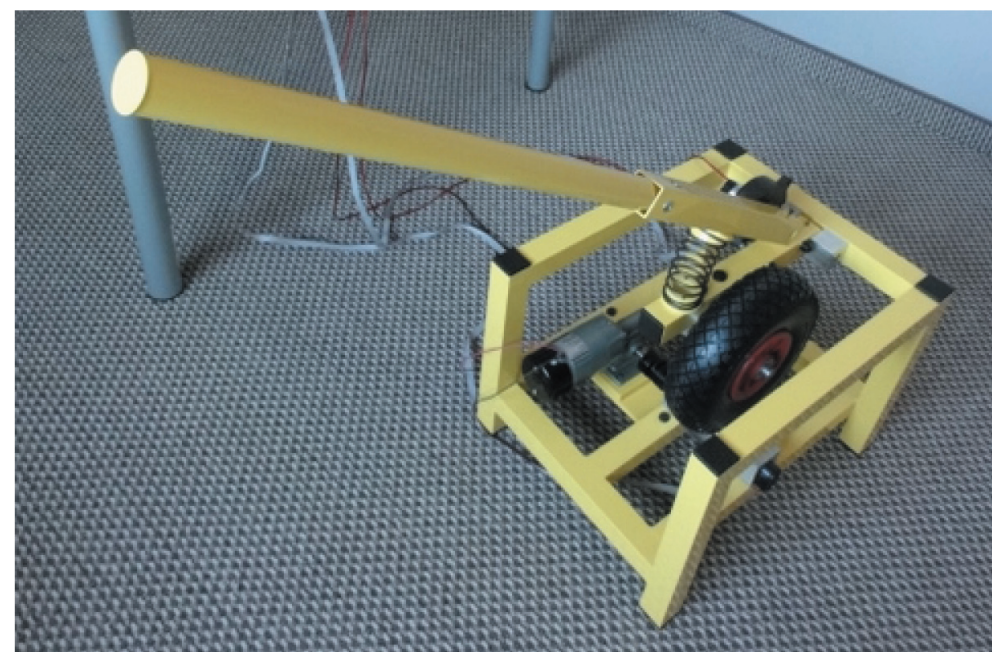

Fig. 1. Photography of the SAS

\subsection{System Overview}

The semi-active suspension laboratory system scheme is depicted in Figure 2. The device consists of three rotating elements: the eccentricity, the unsprung wheel and the sprung body. The small eccentric aluminum wheel is driven by a DC high-torque flat motor with a gear causing vertical kinematic oscillations. The wheel is composed of a plastic rim and a gum tire and is attached to the rigid frame through an arm. It rolls and oscillates due to eccentricity movement. The generated wheel vibrations act on the body arm throughout the suspension system which consists of two elements: a magnetorheological rotary damper and a spring. Resulting vibrations of the body arm depend on the suspension system and the frequency of rotations of the eccentric disk.

The apparatus is equipped with three incremental encoders HEDM-5055 measuring:

- the rotation angle of eccentricity $\alpha_{0}$,

- the rotational angle $\alpha_{1}$ between the wheel arm and the horizontal frame,

- the rotational angle $\alpha_{2}$ between the body arm and the wheel arm.

The encoders resolutions 4096 impulses per revolution (accuracy equal to $0.001534 \mathrm{rad}$ ). These three position sensors signals and corresponding reconstructed angular velocities 
$\left(\dot{\alpha}_{0}, \dot{\alpha}_{1}\right.$ and $\left.\dot{\alpha}_{2}\right)$ constitute system outputs. There are two control pulse width modulation (PWM) input signals:

- DC current,

- MR damper coil current.

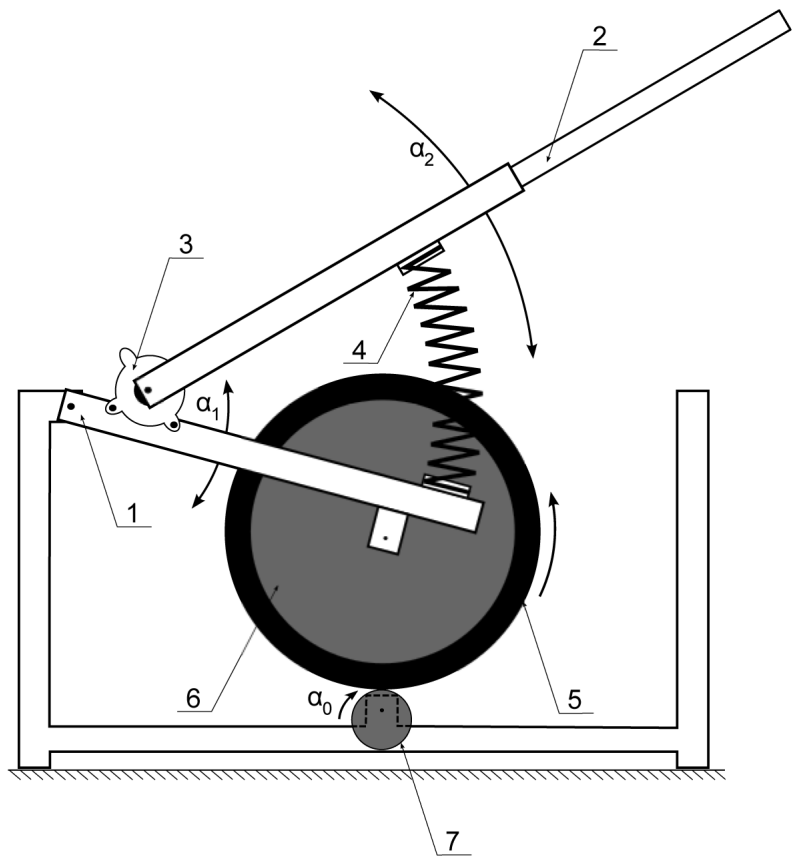

Fig. 2. Scheme of the semi-active suspension system: 1 - wheel arm, 2 - body arm, 3 - MR damper, 4 - spring, 5 - wheel rim, 6 - wheel tire, 7 - eccentric wheel

The first PWM signal is designed to stabilize the rotating frequency of the eccentric disk. Since the DC flat motor is coupled to an eccentric small wheel, the motor load varies with the angular position $\alpha_{0}$ and the suspension system's current state. This has a huge impact on the angular velocity and resulting small eccentric wheel frequency that the $\dot{\alpha}_{0}$ controller is introduced (in detail described in section 2.2). The second PWM control adjusts a MR rotary brake coil current which in effect changes the damping friction coefficient. The SAS system uses to $R D-2087-01$ MR rotary damper manufactured by Lord Corporation [4]. For a detailed brake description please refer to section 2.3. The resolution of both PWM signals is set to 8 bits.

All examined input and output signals are connected to the interface and power supply unit. Control and data acquisition can be performed in several ways:

- dedicated RT-DACI/O board and MATLAB ${ }^{\circledR} /$ Simulnik $^{\circledR} / R T W T$,

- NI Single-Board RIO card and Lab VIEW,

- or Siemens PLC. 
The experiments are conducted in $M A T L A B^{\circledR} /$ Simulnik $^{\circledR}$ real-time environment using the external USBRT-DAC control and measurement board (the PWM control and encoder logics are implemented in a FPGA Xilinx chip). The system sampling period is set to $0.01 \mathrm{~s}$.

The typical system encoder measured angles are shown in Figure 3. The angles $\alpha_{1}$ and $\alpha_{2}$ represent displacement from the steady-state positions for $\alpha_{0}=0$ (as shown in Fig. 2). The eccentric wheel frequency is set to $1.35 \mathrm{~Hz}$ and the MR damper is inactive in this case (input current PWM is set to zero). One can notice an increasing magnitude of angular osciallations of the body. Reducing these osciallations to a minimum via the MR rotary brake control is the goal of the system.
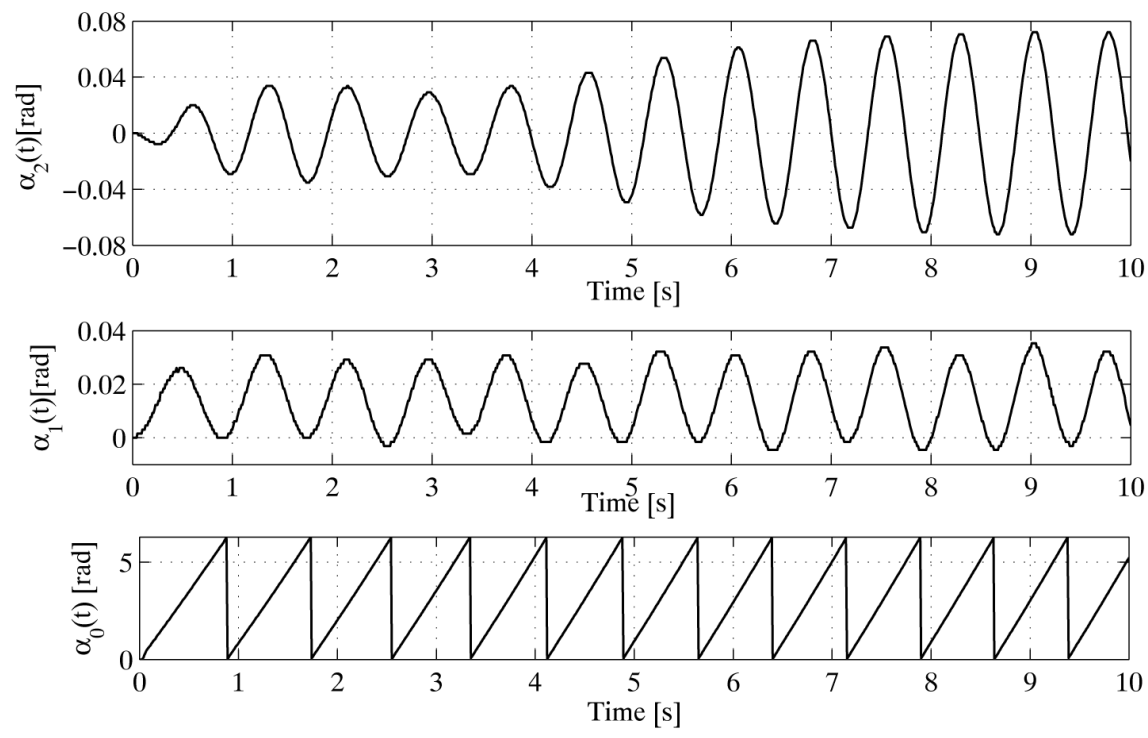

Fig. 3. Angular displacement from a stationary point of body arm $\left(\alpha_{2}\right)$ and wheel arm $\left(\alpha_{1}\right)$ vs. time; eccentricity rotational angle $\alpha_{0}$ vs. time

\subsection{Kinetic excitation controller}

The kinetic excitation controller is applied in the SAS device. It is required due to the eccentric wheel angular velocity variations. A stable eccentricity rotation frequency is necessary for proper model identifiaction and measurement data interpretation. As noticed in section 2.1, eccentric velocity disturbances are produced by a variable load due to the complex suspension structure and the rotational motion itself. In the first half turn (starting from the lowest position) the eccentricity raises the wheel (high load), while during the second half turn the wheel is lowered (small load). As a result, kinetic excitation velocity varies around the value designated from the PWM control.

A frequency controller has been introduced in [3]. Stabilization and compensation of disturbances is based on two inputs: the angular position $\left(\alpha_{0}\right)$ and angular velocity $\left(\dot{\alpha}_{0}\right)$ of 
the eccentric wheel. In fact, the heuristic controller is a combination of position set-point tracking and velocity stabilization controllers (including PID algorithm).

The performance results of the controller operation are shown in Figure 4 and Figure 5. In both figures a solid line indicates the desired frequency value and the dotted line is an instantaneous frequency calculated from the measured eccentric wheel rotational angle.

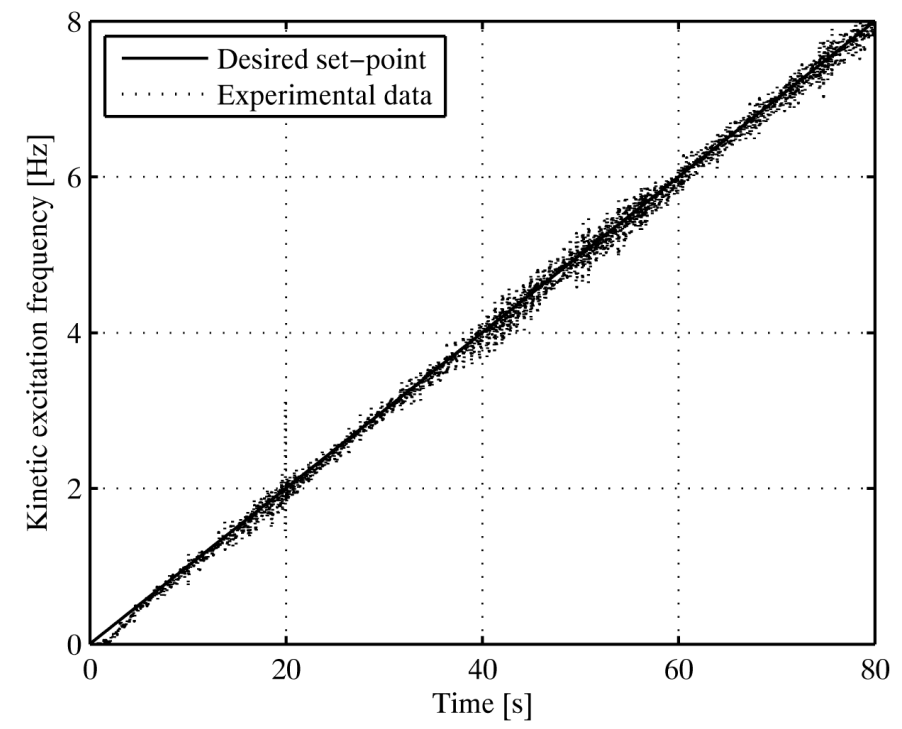

Fig. 4. Set-point (ramp signal) and real measured frequency vs. time

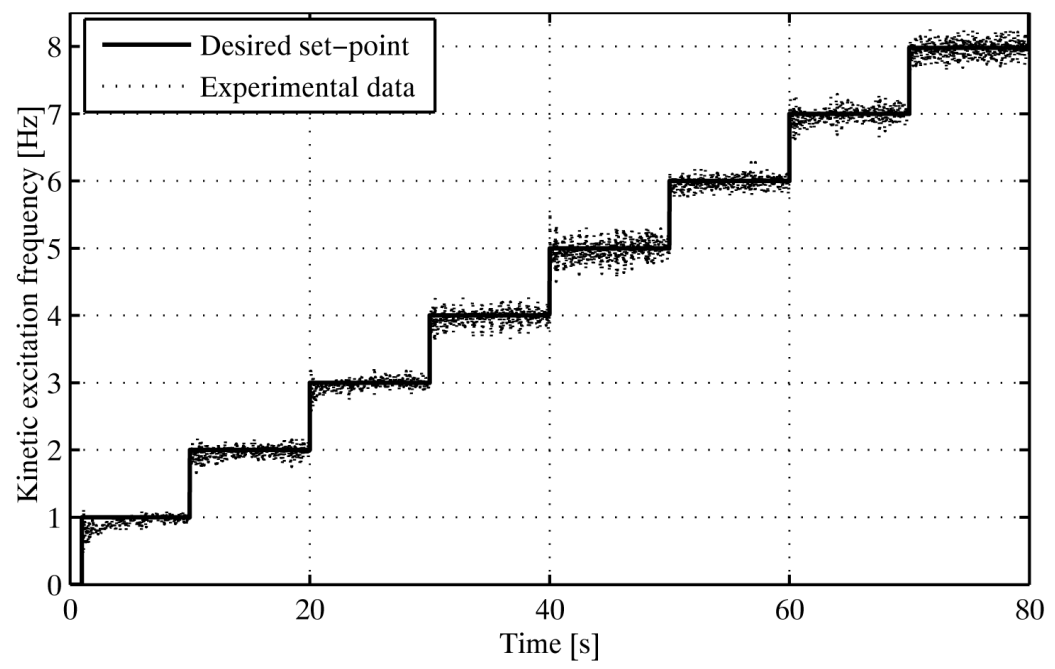

Fig. 5. Set-point (stairs sequence signal) and real measured frequency vs. time 


\subsection{Magnetorheological rotary brake}

The Lord RD-2087-01 rotary brake is a compact, magnetorheological (MR) fluid proportional brake suitable for a wide variety of applications. The magnetorheological fluid (MRF) is a suspension consisting of micron-sized, magnetizable particles and carrier liquid. In the normal condition (where there is no presence of a magnetic field) the fluid is freeflowing liquid. As the magnetic field is applied the particles acquire a dipole moment aligned with the external field that causes particles to form linear chains parallel to the field. In the result the MRF can solidify and restrict its movement ability [8]. The MR dampers use this phenomenon to provide increased torque output. One of the major unit's benefits is the fast response time. The device reacts to the change of input current (PWM duty cycle) in less than 10 milliseconds. This allows for efficient system control, so that body oscillations can be reduced [6].

\section{Modelling and parameter identification}

The semi-active system model identification procedure was divided into two parts: models identification of the suspension and the wheel-eccentricity was conducted separately. Initially, the wheel arm is lifted to the horizontal position and is immobilized. This settlement is suitable to obtain spring and MR damper parameters separately. Afterwards the obtained model is rebuilt to take into account the wheel and the eccentricity. The established wheel lock is removed and missing model parameters are estimated.

\subsection{Suspension part model identification}

\subsubsection{Mathematical model}

The configuration where a wheel arm is locked in the plane position is described in Figure 6 . In this horizontal arrangement the wheel angle $\left(\alpha_{1}\right)$ equals zero and will be considered as ground. A number of variables are introduced:

$r_{s 1}-$ the distance between the spring mount on the wheel arm and the wheel arm axis of rotation,

$r_{s 2}-$ the distance between the spring mount on the body arm and the wheel arm axis of rotation,

$\gamma$ - the angle between the wheel arm (the horizontal line) and $r_{s 2}$.

The nonlinear differential equation used to represent the dynamics is as follows

$$
I \ddot{\gamma}=k_{s} r_{s 2} l_{s}(\gamma)-M \cos (\gamma)-k_{M R}(i) \dot{\gamma}
$$

where

$$
l_{s}(\gamma)=l_{s 0}-\sqrt{r_{s 1}^{2}+r_{s 2}^{2}-2 r_{s 1} r_{s 2} \cos (\gamma)}
$$




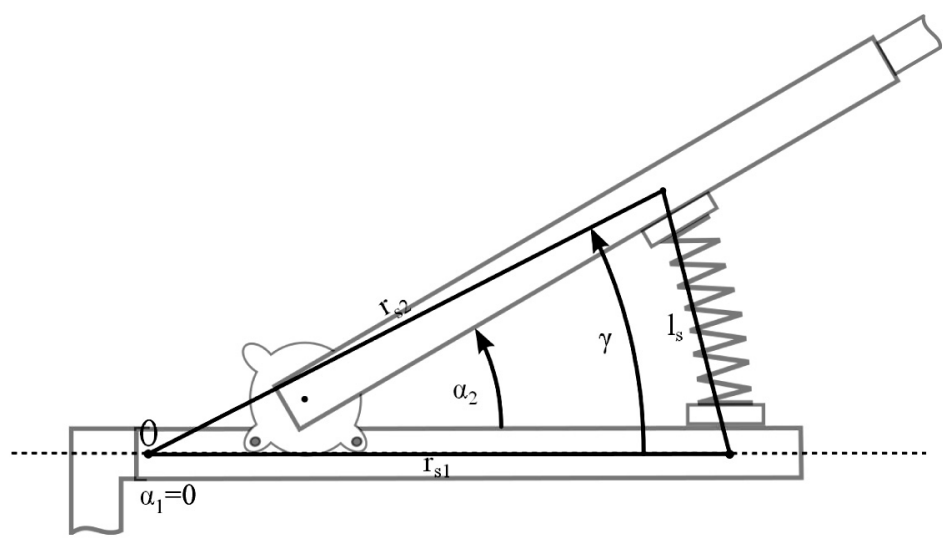

Fig. 6. Structure of the suspension part model

As can be noticed, a few simplifications have been done. The MR damper unit is virtually moved to the wheel arm pivot. Both in the discussed model and in the complete one, the mentioned axis acts as the axis of rotation for the two arms. Without this assumption the model becomes much more complex and a lot of problems may occur (including a variable moment of inertia of the wheel arm or many geometrical issues). The next reduction concerns the spring. The actual length of the spring is calculated with cosine formula (2) using a real $r_{s 1}$, an artificially introduced $r_{s 1}$ and an angle $\gamma$ which slightly differs from the measured body arm angle of rotation $\alpha_{2}$. It is also assumed that the spring obeys Hooke's law ( $k_{s}$ - elasticity coefficient, $l_{s 0}-$ a length of the no-load spring) and exerted restoring force at the end of the spring is perpendicular to $r_{s 2}$. The simplest MR rotary brake parameter model was implemented in [5] where coefficient $k(i)$ is a function of magnetic coil current $i(t)$ and damping is propotional to the angular velocity $\dot{\gamma} . I_{1}$ is the moment of inertia of the wheel arm with respect to the introduced axis of rotation and $M_{1}$ stands for gravitational moment of the body arm.

\subsubsection{Model parameters identification}

There are two primary objectives:

- estimate the suspension part model parameters,

- obtain the best matching MR damper static profile $k_{M R}(i)$.

In the identification procedure the following parameter reduced state equations are considered:

$$
\begin{aligned}
& \dot{x}_{1}=x_{2} \\
& \dot{x}_{2}=a_{1}^{1} l_{s}\left(x_{1}\right)-a_{2}^{1} \cos \left(x_{1}\right)-b x_{2}
\end{aligned}
$$


where

$$
\begin{aligned}
& l_{s}\left(x_{1}\right)=l_{s 0}-\sqrt{r_{s 1}^{2}+r_{s 2}^{2}-2 r_{s 1} r_{s 2} \cos \left(x_{1}\right)} \\
& a_{1}^{1}=\frac{k_{s} r_{s 2}}{I}, \quad a_{2}^{1}=\frac{M}{I}, \quad b=\frac{k_{M R}(i)}{I}
\end{aligned}
$$

The distances $r_{s 1}=0.275[\mathrm{~m}], r_{s 2}=0.191[\mathrm{~m}], l_{s 0}=0.189[\mathrm{~m}]$ and an initial angular position of the body arm $x_{10}=0.57$ [rad] are fixed and their values were measured or calculated directly. Of course the system states denote respectively $x_{1}=\gamma$ and $x_{2}=\dot{\gamma}$.

In the first step a full set of adjustable parameters a $a_{1}^{1}, a_{2}^{1}$ and $b$ have been identified by real-time experiments based on the deflection from the equilibrium angular position $x_{10}$ with inactive MR rotary brake $(i(t)=0[\mathrm{~A}])$. At this point parameter $b$ may be treated as a viscous friction coefficient. Five oscillation trajectories have been collected (enumarated by $i, i=1, \ldots, 5)$ corresponding to different displacements from the steady-state (starting from most significant deflection) and only the first $N=500$ data points are used. Each one consists of the direct measured $\gamma$ and a reconstructed velocity $\dot{\gamma}$ and it is denoted as $x^{i}(n)=\left\{x_{1}^{i}(n), x_{2}^{i}(n)\right\}$ where $n=0,1, \ldots, N$. The identification of parameters is done in MATLAB ${ }^{\circledR}$ System Identification Toolbox and Optimalization Toolbox. Consider the following quality indexes (cost functions):

$$
\begin{aligned}
& Q_{i}\left(x^{i}, \bar{x}\right)=\sum_{j=1}^{2} \sum_{n=1}^{N}\left(x_{j}^{i}(n)-\bar{x}_{j}(n)\right)^{2} \\
& Q(x, \bar{x})=\sum_{i=1}^{5} Q^{i}\left(x^{i}, \bar{x}\right)
\end{aligned}
$$

where $\bar{x}=\left\{x_{1}(n), x_{2}(n)\right\}$ and it is a trajectory generated by the model at the same time instants for a set of parameters $\left\{a_{1}^{1}, a_{2}^{1}, b\right\}$. $x$ denotes merged experiments data points $\left\{x_{1}, x_{2}, \ldots, x_{5}\right\}$. The minimization of the cost functions is performed with respect to the unknown set of parameters:

$$
\begin{aligned}
& Q_{i}^{\text {min }}=\min _{a_{1}^{1}, a_{2}^{1}, b} Q_{i}\left(x^{i}, \bar{x}\right) \\
& Q^{\text {min }}=\min _{a_{1}^{1}, a_{2}^{1}, b} Q\left(x^{i}, \bar{x}\right)
\end{aligned}
$$

The estimation with the quality index in form (6) finds the best matching parameters for a single trajectory contrary to procedure using formula (7) which searches for the unique values common to all experiments. The obtained results are shown in Table 1. 
Table 1

Identified parameters and quality index values

\begin{tabular}{|c|c|c|c|c|c||c|}
\cline { 2 - 7 } \multicolumn{2}{c|}{} & $a_{1}^{1}$ & $a_{2}^{1}$ & $b$ & $Q_{i}^{\min }$ & $Q_{i}$ \\
\hline \multirow{4}{*}{ Experiment $i$} & 1 & 526.61 & 22.07 & 0.20 & 2.40 & 2.44 \\
\cline { 2 - 7 } & 2 & 531.13 & 22.37 & 0.19 & 2.42 & 2.51 \\
\cline { 2 - 7 } & 3 & 535.59 & 22.64 & 0.23 & 2.06 & 3.33 \\
\cline { 2 - 7 } & 4 & 538.56 & 22.76 & 0.29 & 2.00 & 2.76 \\
\cline { 2 - 7 } & 5 & 542.34 & 22.91 & 0.43 & 2.13 & 2.70 \\
\hline \multicolumn{2}{|c|}{ All experiments } & 528.57 & 22.07 & 0.1921 & \multicolumn{2}{c|}{$Q^{\min }=13.74$}
\end{tabular}

$Q_{i}$ are the quality index values calculated for corresponding experiments and obtained parameters set for all real-time trajectories. The identified parameters common to all experiments vary at least from these estimated for trajectories 1 and 2 (the highest displacement from equilibrium point) and deviate at most from the values found for last experiment (the smallest deflection). One can noticed that parameter $a_{2}^{1}$ differs at least among the estimated sets.

The comparision between real-time and commonly modeled trajectories is shown in Figure 7 (experiment 1) and in Figure 8 (experiment 5). Obviously the best fit is attained for more significant magnitude osciallations (similar matching appears in the experiment 2). The less satisfactory results have been achieved near the steady-state position, however since our goal is to use the model to simulate the system behaviour nearby the resonant frequency, where magnitude of oscillations rapidly increases, this inaccuracy is acceptable.

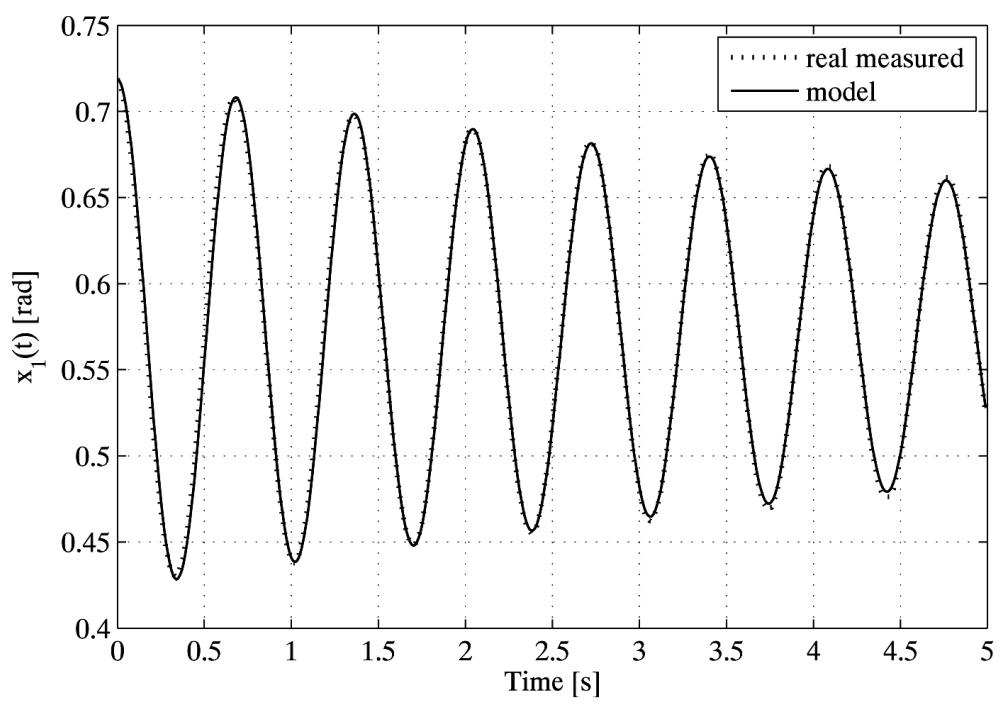

Fig. 7. Body angular position $x_{1}$ vs. time in experiment 1 


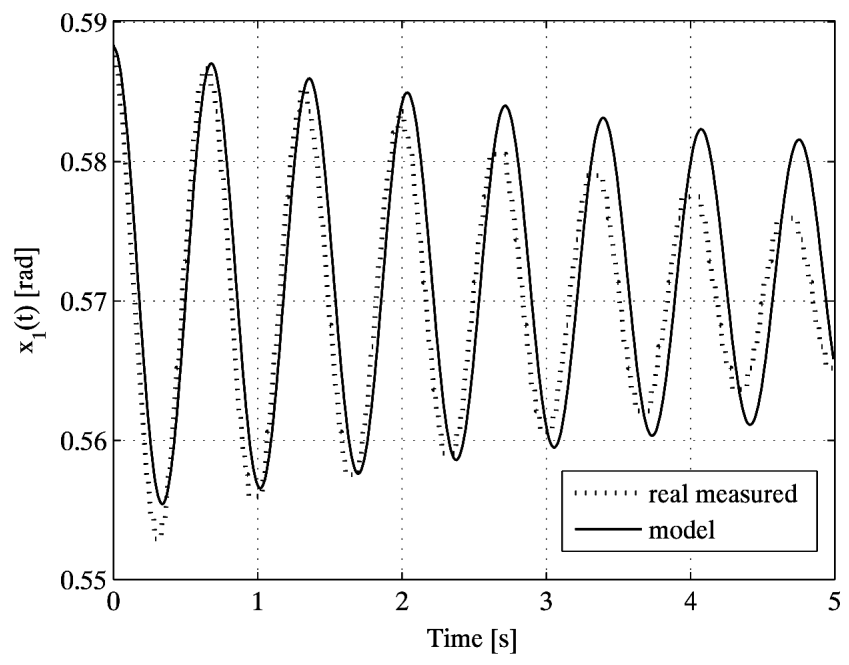

Fig. 8. Body angular position $x_{1}$ vs. time in experiment 5

Having the model parameters $a_{1}^{1}$ and $a_{2}^{1}$ identified, the MR brake static profile can be obtained. The same mathematical model (3) is used but the mentioned parameters $a_{1}^{1}$ and $a_{2}^{1}$ are fixed. In this step the body arm is deflected by approximately the same value from the steady-state angle however each time the different magnetic coil current $i(t)$ is applied. Twenty-one trajectories have been collected corresponding to the input current PWM duty cycles $u_{M R}=0.05(i-1)$ where $i=1,2, \ldots, 21$. Since the oscillation cycles number decreses due to the higher values of $u_{M R}$ the experiment data samples number changed from 2700 to 200 and is denoted $N_{i}$. The cost function takes the form:

$$
Q_{i}\left(x^{i}, \bar{x}\right)=\sum_{j=1}^{2} \sum_{n=1}^{N}\left(x_{j}^{i}(n)-\bar{x}_{j}(n)\right)^{2}
$$

and the minimalization is performed for each $i$ with respect to an unknown parameter $b$ :

$$
Q_{i}^{\min }=\min _{b} Q_{i}\left(x^{i}, \bar{x}\right)
$$

The obtained static profile of the magnetorheological rotary brake as a damping coefficient function of $u_{M R}$ is depicted in Figure 9. The visible points indicates estimated values band the solid line is a continous function (linear interpolation) introduced to test the model in the form:

$$
\begin{aligned}
& \dot{x}_{1}=x_{2} \\
& \dot{x}_{2}=a_{1}^{1} l_{s}\left(x_{1}\right)-a_{2}^{1} \cos \left(x_{1}\right)-b\left(u_{M R}\right) x_{2}
\end{aligned}
$$

The verification procedure of the model (12) is based on two real-time experiments. The results are shown in Figure 10 and Figure 11. The figures illustrate the decreasing body 
angular position oscillations due to the different $u_{M R}$ signal. The real measured vibrations are marked with the dotted line and the vibrations obtained from the model with the solid line. As can be noted, in general modeled trajectories match real data points. The constantly visible error at the end of the motion is acceptable as mentioned above and can be justified by the implemented model simplifications.

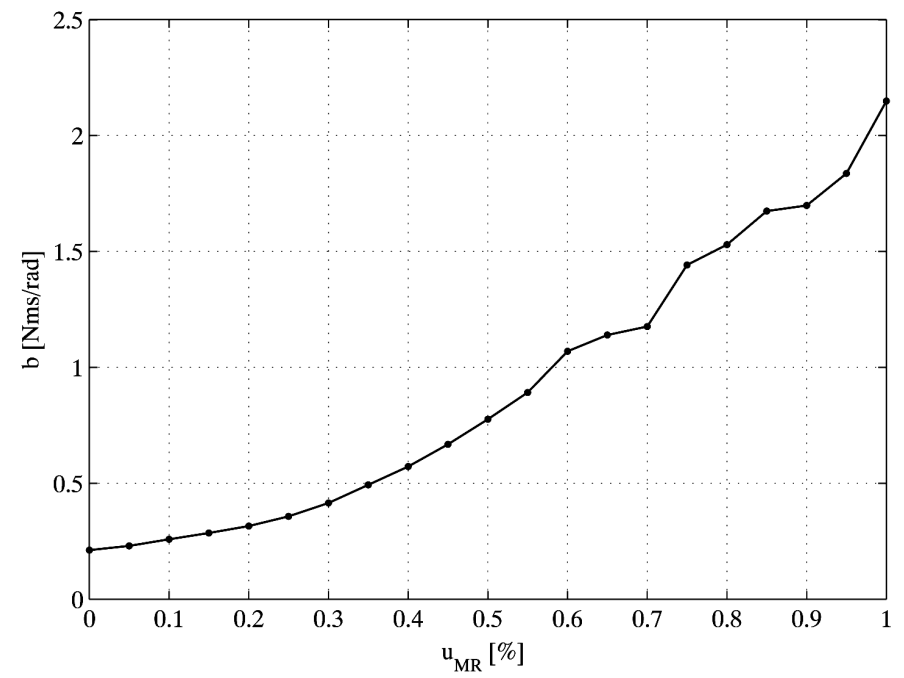

Fig. 9. Obtained static profile $b\left(u_{M R}\right)$ of MR damper
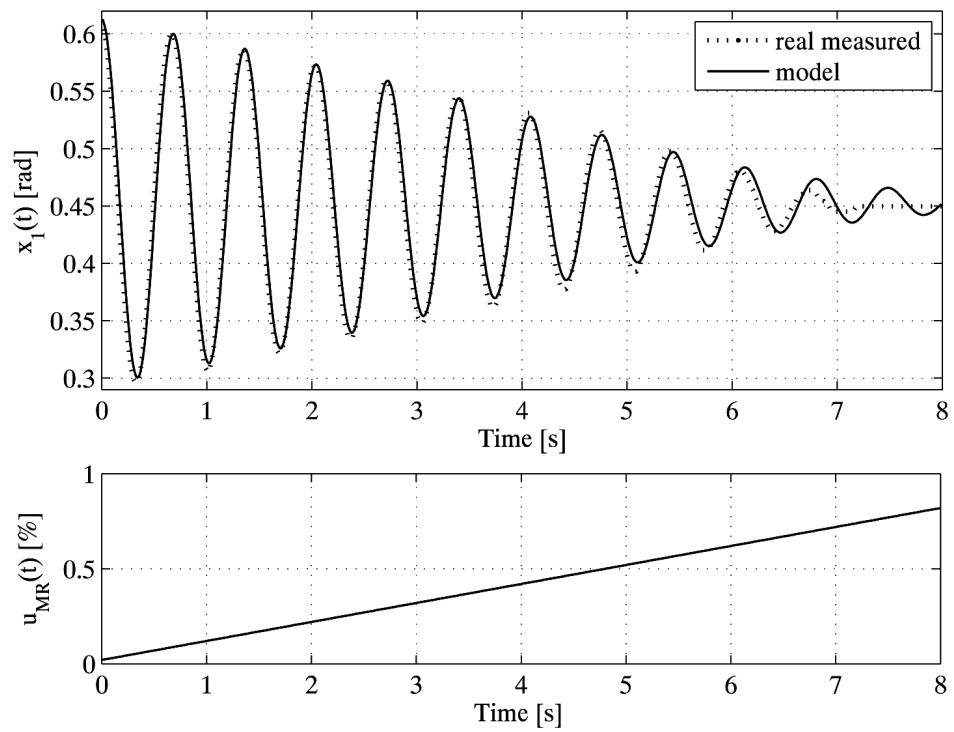

Fig. 10. Body angle $x_{1}$ and MR damper control $u_{M R}$ vs. time 

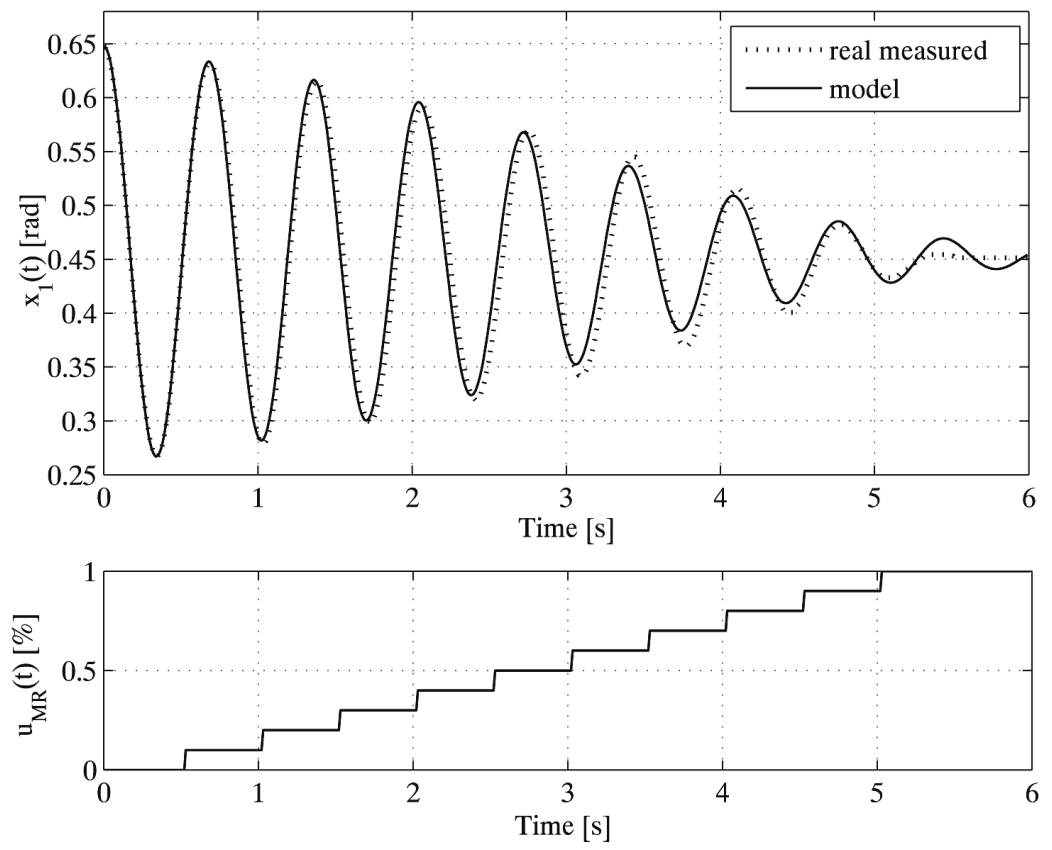

Fig. 11. Body angle $x_{1}$ and MR damper control $u_{M R}$ vs. time

At this point with the parameters and the MR static profile obtained one can compute the value of $k_{s}$. The rotational inertia $I$ is set to $1.37\left[\mathrm{~kg} \cdot \mathrm{m}^{2}\right]$ and it is calculated using the formula for the moment of inertia of a rod with respect to the axis of rotation at the end of the bar. In the result $k_{S}=3.79 \cdot 10^{3}\left[\mathrm{~kg} / \mathrm{s}^{2}\right]$ and the MR static profile is muliplied by the value $I$ and labeled as $k_{M R}\left(u_{M R}\right)$.

\subsection{Identification of the wheel-eccentricity part model}

\subsubsection{Mathematical model}

The laboratory device diagram in an natural initial position is shown in Figure 12. The system of ordinary differential equations (ODE) representing the complete mathematical model is an expanded version of (1) with a number of changes as follows:

$$
\begin{aligned}
I_{1} \ddot{\alpha}_{1} & =k_{t} R \cos \left(\beta-\alpha_{1}\right) l_{t}\left(u_{1}, \alpha_{1}\right)+b_{t}\left(\dot{u}_{1}-\dot{\alpha}_{1} R \cos \left(\beta-\alpha_{1}\right)\right)- \\
& -k_{s} r_{s 1} l_{s}\left(\alpha_{1}, \gamma\right)-M_{1} \cos \left(\beta-\alpha_{1}\right)+k_{M R}\left(u_{2}\right)\left(\dot{\alpha}_{1}-\dot{\gamma}\right) \\
I_{2} \ddot{\gamma}= & k_{s} r_{s 2} l_{s}\left(\alpha_{1}, \gamma\right)-M_{2} \cos (\gamma)+k_{M R}\left(u_{2}\right)\left(\dot{\alpha}_{1}-\dot{\gamma}\right)
\end{aligned}
$$


where

$$
\begin{aligned}
& l_{s}\left(\alpha_{1}, \gamma\right)=l_{s 0}-\sqrt{r_{s 1}^{2}+r_{s 2}^{2}-2 r_{s 1} r_{s 2} \cos \left(\gamma-\alpha_{1}\right)} \\
& l_{t}\left(u_{1}, \alpha_{1}\right)=l_{t 0}-\left(H-r-u_{1}-R \sin \left(\beta-\alpha_{1}\right)\right)
\end{aligned}
$$

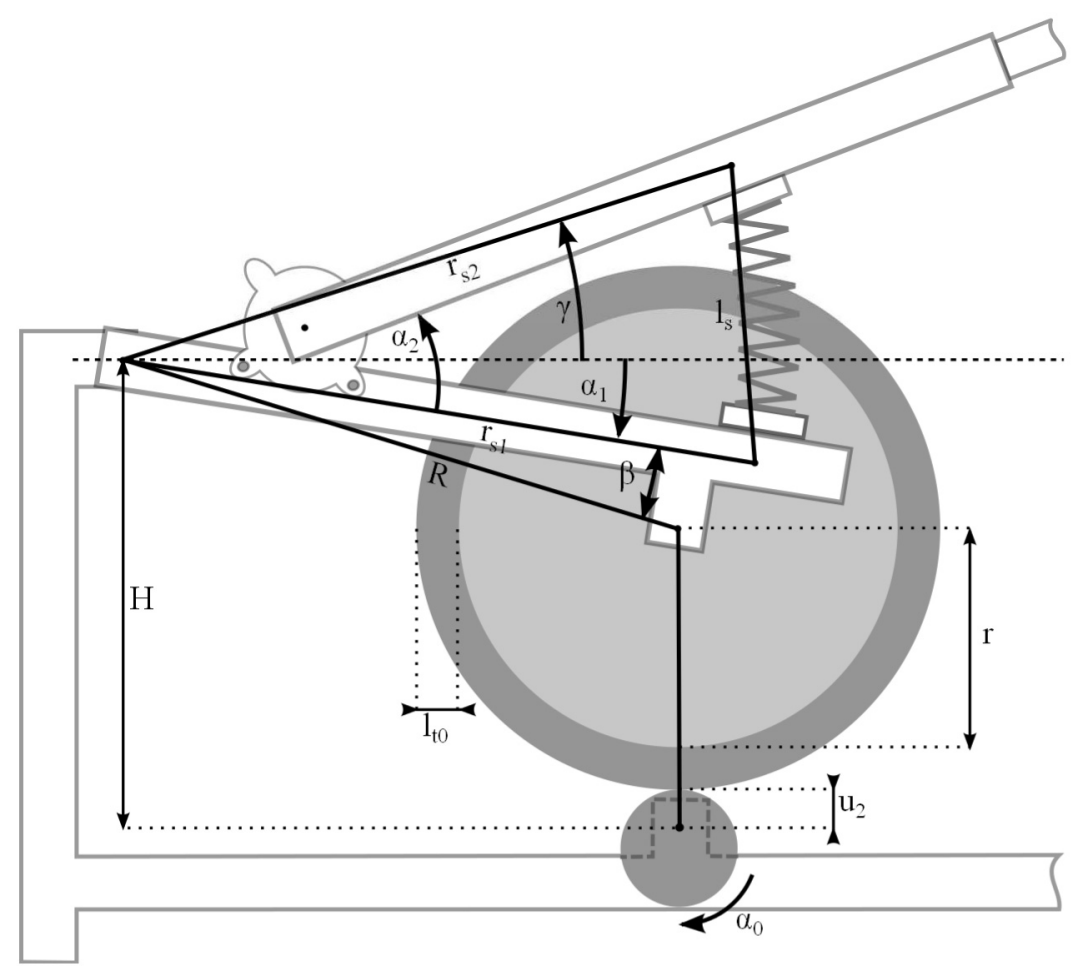

Fig. 12. Structure of the complete SAS model

The second equation in (13) describing the dynamics of the body arm compared to equation (1) differs in a spring length calculation manner (the wheel arm rotates, consequently $\alpha_{1} \neq 0$ ) and the static profile of the MR brake is multiplied by the angular velocities difference. The more complex first equation includes new distances:

$R$ - the wheel center rotation radius with respect to the pivot 0 ,

$H$ - the distance between the pivot 0 and the pivot of the eccentric wheel,

$r$ - the radius of the wheel rim,

$l_{t 0}-$ the thickness of the no-load wheel tire.

The fixed angle between $R$ and $r_{s 1}$ is denoted by $\beta$. Since $\alpha_{1}$ is concerned to be expressed in negative radians then obviously $\beta-\alpha_{1}$ is the angle between the wheel center (also 
considered as the wheel mass center) rotation radius and the horizontal line. Of course, the values $I_{1}$ and $I_{2}$ stand for wheel and body arm moments of inertia. Again, $M_{1}$ and $M_{2}$ are gravitational moments of respectively wheel and body equivalent mass. Of course the spring force moment also occurs in the wheel motion equation as well as the MR damper torque.

There are two inputs $u_{1}$ and $u_{2}$. The second signal equals the PWM duty cycle $u_{M R}$ (renamed due to matching final model consequent terminology), while

$$
u_{1}=u_{1}\left(\alpha_{0}\right)=0.0425-0.0025 \cos \left(\alpha_{0}\right)
$$

is a distance between the eccentric pivot and an eccentricity upper level (see Fig. 12). As one can notice that lowest level equals to $u_{2}\left(\alpha_{0}=0\right)=0.04[\mathrm{~m}]$ and this eccentric wheel configuration is considered as an initial position (all experiments are started from there). Indeed the excitation generated by the eccentric wheel is not exactly in the form of a given cosine function but it can be approximated using this formula particularly for constant frequencies.

As can be noted, the interaction between the eccentric and the wheel is modeled as a standard mass-spring-damper system $\left(k_{t}\right.$ denotes a tire resilience coefficient and $b_{t}$ is an absorption coefficient of the tire). This approach requires additional model simplifications. It is assumed that the wheel tire does not slip on an eccentric wheel during its rotations and furthermore that the wheel is always 'coupled' with the eccentricity (does not hop). The described behaviour is present in the system (sliding is observed for higher eccentric velocities and the wheel jumping occurs near body resonant frequency). However, unless the model is dedicated to the lower eccentric frequency range, it can be successfully applied to reduce body oscillations which is the goal of the controller.

\subsubsection{Model parameters identification}

As in section 3.1.2 the identification process is performed using parameter reduced state equations in a form:

$$
\begin{aligned}
\dot{x}_{1} & =x_{2} \\
\dot{x}_{2} & =a_{1}^{2} \cos \left(\beta-x_{1}\right) l_{t}\left(u_{1}, x_{1}\right)+a_{2}^{2}\left(\dot{u}_{1}-x_{2} R \cos \left(\beta-x_{1}\right)\right)- \\
& -a_{3}^{2} \cos \left(\beta-x_{1}\right)+k_{1}^{2} k_{M R}\left(u_{2}\right)\left(x_{2}-x_{4}\right)-a_{4}^{2} l_{s}\left(x_{1}, x_{2}\right) \\
\dot{x}_{3} & =x_{4} \\
\dot{x}_{4} & =a_{5}^{2} l_{s}\left(x_{1}, x_{3}\right)-a_{6}^{2} \cos \left(x_{3}\right)+k_{2}^{2} k_{M R}\left(u_{2}\right)\left(x_{2}-x_{4}\right)
\end{aligned}
$$


where

$$
\begin{aligned}
& l_{s}\left(x_{1}, x_{3}\right)=l_{s 0}-\sqrt{r_{s 1}^{2}+r_{s 2}^{2}-2 r_{s 1} r_{s 2} \cos \left(x_{3}-x_{1}\right)} \\
& l_{t}\left(u_{1}, x_{1}\right)=l_{t 0}-\left(H-r-u_{1}-R \sin \left(\beta-x_{1}\right)\right) \\
& a_{1}^{2}=\frac{k_{t} R}{I_{1}}, \quad a_{2}^{2}=\frac{b_{t}}{I_{1}}, \quad a_{3}^{2}=\frac{M_{1}}{I_{1}} \\
& a_{4}^{2}=\frac{k_{s} r_{s 1}}{I_{1}}, \quad a_{5}^{2}=\frac{k_{s} r_{s 2}}{I_{2}}, \quad a_{6}^{2}=\frac{M_{2}}{I_{2}} \\
& k_{1}^{2}=\frac{1}{I_{1}}, \quad k_{2}^{2}=\frac{1}{I_{2}}
\end{aligned}
$$

The state variables are adopted as follows: $x_{1}=\alpha_{1}, x_{2}=\dot{\alpha}_{1}, x_{3}=\gamma$ and $x_{4}=\dot{\gamma}$. The initial angular position of the wheel arm is obtained through the encoder indications, calculated as a difference between the wheel arm horizontal alignment and the normal position where the wheel lies on the eccentricity. In the result, the measured value $x_{10}$ equals -0.19 [rad]. To determine the initial angle of the body arm $x_{30}$ in the model (13) the following steps are taken:

- the spring length calculation equation (4) in the model (3) is modified and takes the form:

$$
l_{s}\left(x_{1}\right)=l_{s 0}-\sqrt{r_{s 1}^{2}+r_{s 2}^{2}-2 r_{s 1} r_{s 2} \cos \left(x_{1}-\theta\right)}
$$

- the introduced $\theta$ is set to the wheel arm initial angle $x_{10}$,

- the simulation of the revised model with previously identified parameters (5) is performed (starting from the formerly steady-state position),

- the obtained equilibrium of the system is considered as $x_{30}$ and equals approximately 0.36 [rad].

The distances $R, H, r$ and $l_{t 0}$ as well as the angle $\beta$ are preliminary measured, although only the last three values are fixed from the beginning. Since the tire stiffness is large it is difficult to precisely measure loaded tire thickness (expressed as $\left(H-r-u_{1}-R \sin \left(\beta-x_{1}\right)\right.$ in the formula (18)) in the system steady-state position. Instead, this value has been set to 0.0185 [m] (that is 0.0015 [m] less than no-load tire thickness) and distances $R$ and $H$ are adjusted using simple optimization procedure in such a way that:

$-l_{t}\left(u_{1 \min }=u_{1}(0), x_{10}\right)=0.0015$ and

$-l_{t}\left(u_{1 \max }=0.045, x_{1}=-0.167\right)$ equals approximately to 0.002 .

The angle $x_{1}=-0.167$ is the encoder measured position of the wheel arm for the highest eccentric wheel level and the value 0.002 is introduced in order to guarantee that 
the rubber tire is insignificantly more squeezed in the second case. The obtained values of $R$ and $H$ only slightly differ from measured lengths and finally the following fixed variables are used in the model (16): $R=0.21[\mathrm{~m}], H=0.22[\mathrm{~m}], r=0.075[\mathrm{~m}], l_{t 0}=0.02[\mathrm{~m}]$ and $\beta=0.234$ [rad].

In order to obtain a set of final model parameters $p=\left\{a_{1}^{2}, a_{2}^{2}, a_{3}^{2}, a_{4}^{2}, a_{5}^{2}, a_{6}^{2}, k_{1}^{2}, k_{2}^{2}\right\}$ one real-time experiment is conducted. An experiment is performed for a ramp frequency signal applied to a kinetic excitation controller $\left(f_{u 1}(t)=0.1 t[\mathrm{~Hz}]\right)$. The fitting pattern is considered as first $N=2000$ data points. The PWM control signal $u_{2}$ of the MR rotary brake is set to zero. The values $u_{1}(n)$ where $n=1,2, \ldots, N$ denote the control calculated using formula (15) from a rotation angle $\alpha_{0}(n)$ of the eccentricity. The corresponding trajectory $x(n)=\left\{x_{1}(n), x_{2}(n), x_{3}(n), x_{4}(n)\right\}$ consist of:

- a direct measured $x_{1}=\alpha_{1}$,

- a reconstructed velocity $x_{2}=\dot{\alpha}_{1}$,

- an indirect measured $x_{3}=\alpha_{2}+\alpha_{1}$ (see Fig. 12) and

- a velocity $x_{4}=\dot{\alpha}_{2}+\dot{\alpha}_{1}$.

Similarly, quality index takes a form:

$$
Q(x, \bar{x})=\sum_{j=1}^{4} \sum_{n=1}^{N}\left(x_{j}(n)-\bar{x}_{j}(n)\right)^{2}
$$

where $\bar{x}=\left\{x_{1}(n), x_{2}(n), x_{3}(n), x_{4}(n)\right\}$ is a trajectory obtained for parameters $p$.

Again, the cost functions minimization is performed with respect to the unknown parameters $\tilde{p}$ :

$$
Q^{\min }=\min _{\tilde{p}} Q(x, \bar{x})
$$

where $\tilde{p}$ is a subset of the model parameters $p$. Several selecting approaches as to which parameters to fix and which to estimate have been considered. Of course, the values of $a_{5}^{2}$ and $a_{6}^{2}$ are substituted with the previously identified parameters $a_{1}^{1}$ and $a_{2}^{1}$ as well as $k_{2}^{2}$ is set to the inverse value of the adopted $I=I_{2}$ in section 3.1.2. As it was noted, without further fixings the optimization procedure tends to find parameters which match a pattern well, however, their values do not have a physical meaning. In the course of the research it has been proven that making additional parameters constant during the estimation process does not decrease the quality of the model. In fact, the identified values have a relation to the reality. Initially the moment of inertia of the wheel arm $I_{1}$ is set to the value 0.2116 calculated using the known formula $I_{1}=m_{1} R^{2}$ (the wheel mass $m_{1}$ is considered as the point mass at the distance $R$ from the axis of rotation) hence $k_{1}^{2}=4.72$. Having $I_{1}$ we can also determine $a_{4}^{2}=4.92 \cdot 10^{3}$ using formerly estimated $k_{S}$. The last fixed parameter is $a_{3}^{2}=\frac{m_{1} g R}{I_{1}}=46.71$, where $g=9.81\left[\mathrm{~m} / \mathrm{s}^{2}\right]$ stands for gravitational acceleration. After 
determining the fixed values of the model parameters described above the estimating subset $\tilde{p}$ becomes $\left\{a_{1}^{2}, a_{2}^{2}\right\}$.

In the result of the quality index (21) minimization, the following parameters are identified: $a_{1}^{2}=1.08 \cdot 10^{5}, a_{2}^{2}=1.44 \cdot 10^{3}$ and the corresponding cost function value equals $Q^{\min }=34.39$. The estimation procedure has been repeated several times to ensure that the minimum is not a local one. The obtained model steady-state is slightly different from initially adopted and equals $x_{0}=\left[\begin{array}{llll}-0.195 & 0 & 0.3540\end{array}\right]^{T}$. The real-time experiment utilized as a pattern in the identification procedure has been transformed to take into account the new system equilibrium position and it is compared to the obtained modeled trajectory in Figure 13. As can be noted, the both model states trajectories $x_{1}$ and $x_{3}$ (solid line) fit the experimental data (dotted line) well in the most time instants. In fact, the model behavior is also correct beyond the range of frequencies used in the identification (Fig. 14).
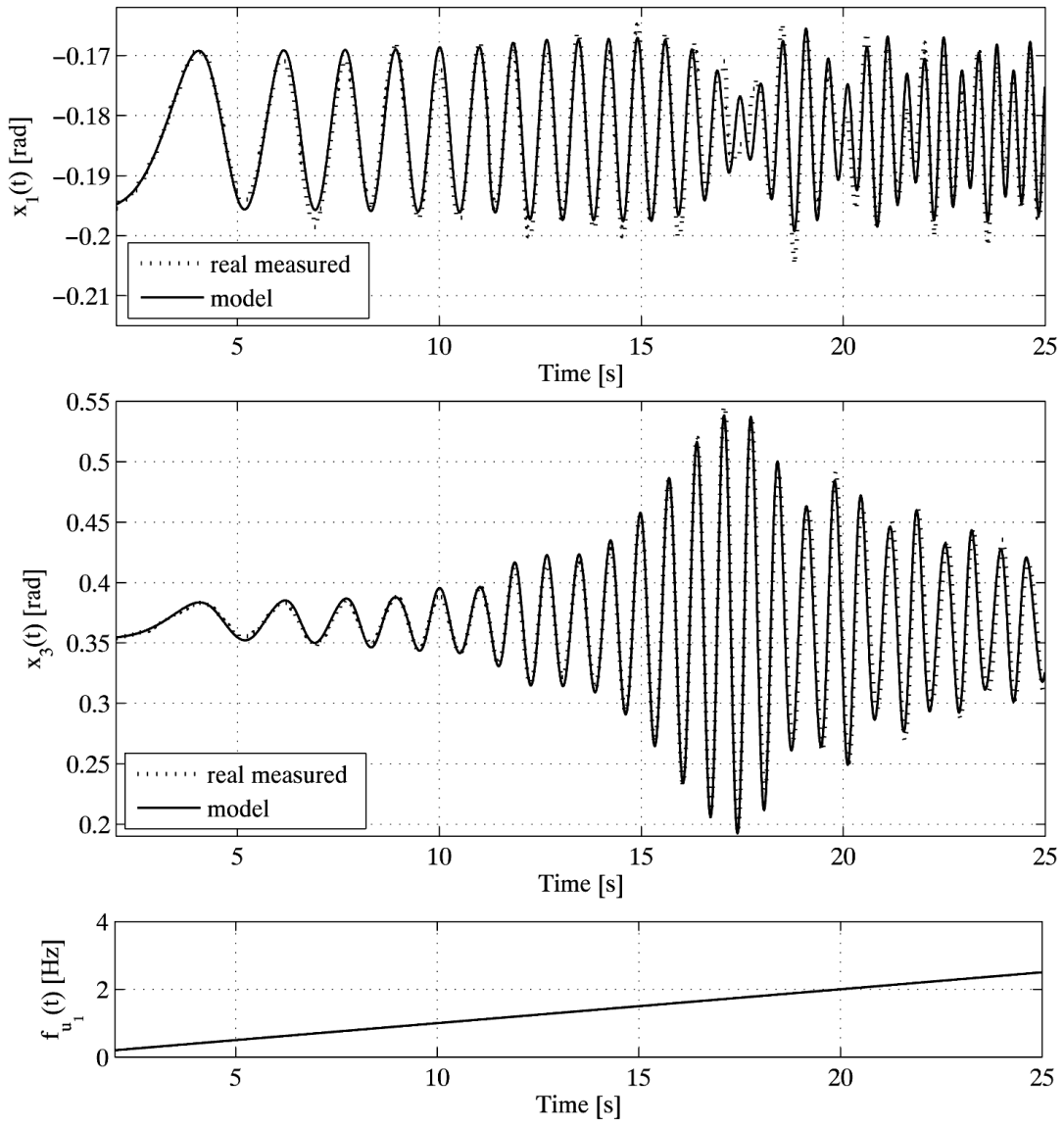

Fig. 13. Wheel angular position $x_{1}$, body angular position $x_{3}$ and set-point frequency for the kinetic excitation controller $f_{u_{1}}$ vs. time 

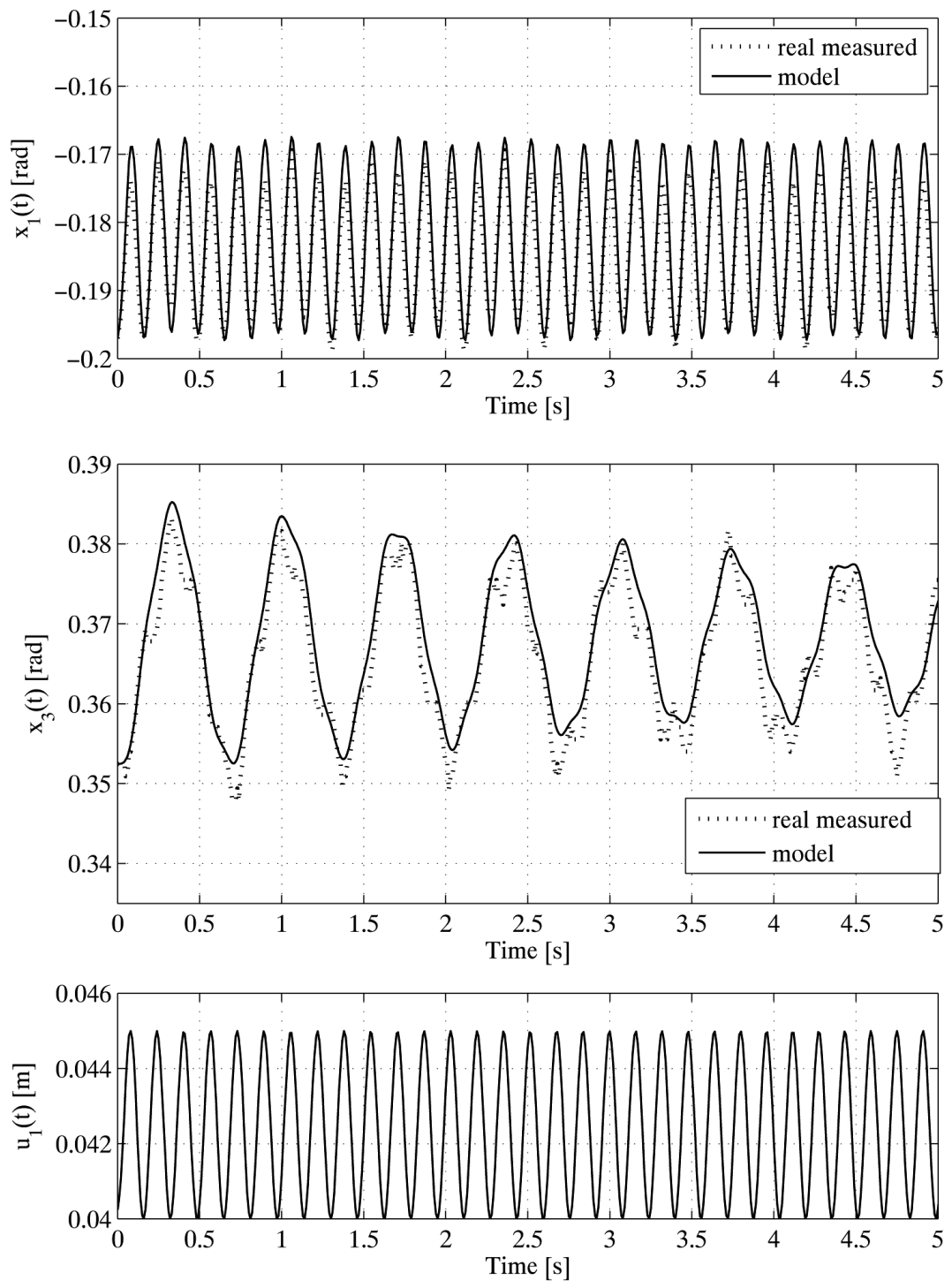

Fig. 14. Wheel angular position $x_{1}$, body angular position $x_{3}$ and control $u_{1}$ vs. time $\left(f_{u_{1}}(t)=6.5[\mathrm{~Hz}]\right)$

Having tha last two parameters identified we can calculate the missing parameters $k_{t}=\frac{a_{1}^{2} I_{1}}{R}=1.09 \cdot 10^{5}$ and $b_{t}=a_{2}^{2} I_{1}=305.27$. At this point the complete model is identified and the last action is to test and verify the obtained static profile of the MR damper. Initially, the system behaviour due to different $u_{2}$ values has been tested in a series of experiments similar to the one conducted in section 3.1.2 during the profile determination. As was 
expected the modelled and experimental trajectories are similar. The second verification test is based on the research of the model reactions to variable $u_{2}$ while the eccentricity rotates with constant frequency. Figures 15, 16 and 17 show the model performance for $f_{u_{1}}(t)=1.6[\mathrm{~Hz}]$ and the MR damper control values $u_{2}$ in order $0.3,0.7$ and 1 in the steadystate. In this case the model quality is less satisfactory however acceptable. The differences in the body osciallations amplitude between modelled (solid line) and the measured values (dotted line) can be explained by the model simplifications made (mainly by the simple model of the MR brake). In Figure 17 we can also observe the MR damper's impact on the body vibration frequency.

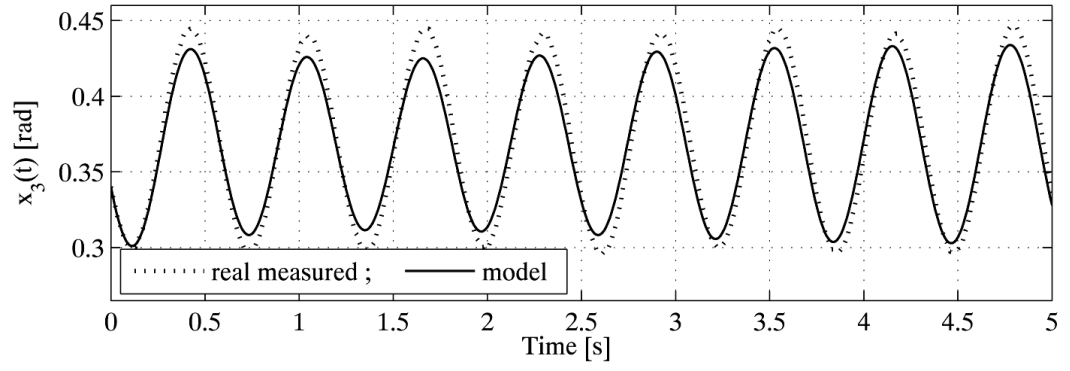

Fig. 15. Body angular position $x_{3}$ vs. time $\left(f_{u_{1}}(t)=1.6[\mathrm{~Hz}], u_{2}=30[\%]\right)$

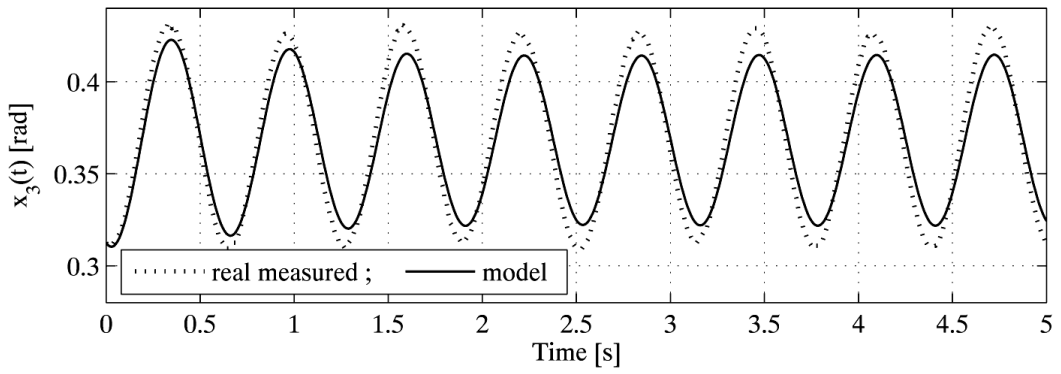

Fig. 16. Body angular position $x_{3}$ vs. time $\left(f_{u_{1}}(t)=1.6[\mathrm{~Hz}], u_{2}=70[\%]\right)$

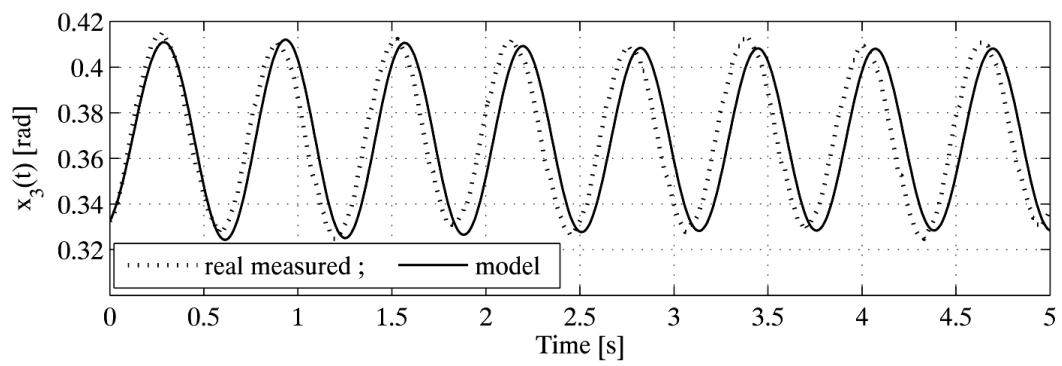

Fig. 17. Body angular position $x_{3}$ vs. time $\left(f_{u_{1}}(t)=1.6[\mathrm{~Hz}], u_{2}=100[\%]\right)$ 


\section{Conclusions}

The described approach to SAS system modelling and the parameters identification technique yields proper and satisfactory results. In spite of mathematical model simplifications with respect to the complicated mechanical structure, the modelled trajectories match real-time data experiments. In order for the model to better reflect body arm oscilations, in respect to MR brake control, a more complicated or even a dynamic MR damper model needs to be used. The laboratory device and the obtained mathematical model can be used to rapidly develop numerous control algorithms which can be easily simulated and tested in real-time experiments.

\section{References}

[1] Eykhoff P., System identification: parameter and state estimation. Wiley, London, 1974.

[2] Gershenfeld N., Mathematical Modeling. Cambridge University Press, United Kingdom, Cambridge, 1999.

[3] Gorczyca P., Kogut K., Kołek K., Rosół M., Turnau A., Wymuszenie kinematyczne zawieszenia pojazdu - kinematic excitation of the vehicle suspension. [in:] L. Trybus and S. Samolej, eds, Projektowanie, analiza i implementacja systemów czasu rzeczywistego. Wydawnictwa Komunikacjii Łączności, Warszawa, 2011.

[4] LORD Corporation. Manual. http://www.lord.com, 2012 [Online].

[5] Sapiński B., Linearized characterization of a magnetorheological fluid damper. Mechanics, 24(2), 2005, 144-149.

[6] Sapiński B., Magnetorheological Dumpers in Vibration Control. AGH University of Science and Technology Press, Cracow, 2006.

[7] Turnau A., Model identification dedicated to the time-optimal control. 17th IFAC World Congress, 7-11 July, Seoul, 2008, 2655-2660.

[8] Wang D.H., Liao W.H., Magnetorheological fluid dampers: a review of parametric modelling. Smart materials and structures, 20, 2011.

[9] Yue C., Control law designs for active suspensions in automotive vehicles. Master's thesis, Massachusetts Institute of Technology, 1987. 